\title{
A Multicriteria Decision Model for Assessment of Failure Consequences in the RCM Approach
}

\author{
Marcelo H. Alencar and Adiel T. de Almeida \\ Universidade Federal de Pernambuco, Cx. Postal 7462, 50.630-970 Recife, PE, Brazil \\ Correspondence should be addressed to Marcelo H. Alencar; marcelohazin@gmail.com
}

Received 2 April 2015; Accepted 4 June 2015

Academic Editor: Huaguang Zhang

Copyright (C) 2015 M. H. Alencar and A. T. de Almeida. This is an open access article distributed under the Creative Commons Attribution License, which permits unrestricted use, distribution, and reproduction in any medium, provided the original work is properly cited.

\begin{abstract}
This paper proposes a multicriteria decision model based on MAUT (Multiattribute Utility Theory) incorporated into an RCM (Reliability Centered Maintenance) approach in order to provide a better assessment of the consequences of failure, allowing a more effective maintenance planning. MAUT provides an evaluation of probability distributions on each attribute as well as trade-offs involving lotteries. The model proposed takes advantage of such evaluations and it also restructures consequence groups established in an RCM approach into new five dimensions. As a result, overall indices of utility are computed for each failure mode analyzed. With these values, the ranking of the alternatives is established. The decision-maker's preferences are taken into account so that the final result for each failure mode incorporates subjective aspects based on the decision-maker's perceptions and behavior.
\end{abstract}

\section{Introduction}

Chouikhi et al. [1] state that, currently, organizations need to comply with environmental protection standards. Production systems of goods and services are subject to random deterioration arising from age and use. The impact of deterioration not only affects the product or service quality but also affects the environment itself.

Ruiz-Castro [2] points out that, in many situations, failures in critical systems generate considerable damage and financial losses resulting from a very low reliability. According to Cavalcante and de Almeida [3], in production systems of goods, losses associated with the downtime of machines can be mitigated by increasing production beyond the normal capacity or adopting some action that can prevent downtime. Ishizaka and Nemery [4] argue that if machines are neglected, this can lead to breakdowns and therefore interruptions to production or the supply of services, delays, scheduling problems, bottlenecks, high costs, and even deaths or injuries.

In this context, Liu et al. [5] argue that maintenance plays an important role in industrial production, especially in areas where the most significant losses arise when a system fails. Ruiz-Castro [2] claims that, in this sense, regular maintenance policies are employed in order to reduce damage. To improve reliability, it is necessary to maintain systems, thereby preventing failures and raising the chance of profit.

However, according to Moubray [6], maintenance has changed over the past decades due to the increase in the number and variety of assets that must be maintained. The biggest challenge today for companies, with respect to maintenance, is to decide what techniques should be used in their organizations, in order to raise the performance of assets and to reduce maintenance costs. According to Kontrec et al. [7], the main goal of maintenance techniques is to ensure that functions of the system are performed in accordance with established standards.

In this context, Selvik and Aven [8] emphasize that planning preventive maintenance activities for technical systems is a constant challenge. On the one hand, a balance is sought between the frequency and extent of maintenance. On the other hand, cost control is pursued. Preventive maintenance is introduced to prevent the occurrence of system failures and to reduce the potential consequences of failures. However, maintenance may also, sometimes, introduce failures. All 
these aspects are relevant for planning preventive maintenance. According to Wang et al. [9], to repair or prevent these failures many maintenance strategies have emerged gradually over time. One of the most relevant ones is RCM (Reliability Centered Maintenance).

This paper will focus on a specific point in the RCM approach: the consequences of failure from the perspective of a multicriteria approach. A multicriteria approach can be found in other studies in the area but is used for different purposes: Chan and Prakash [10] present a fuzzy MCDM approach for maintenance policy selection in manufacturing firms. Kumar and Maiti [11] use a fuzzy analytic network process to deal with the problem of maintenance policy selection for an industrial unit. de Almeida [12] proposes a multicriteria decision model based on MAUT for selecting preventive maintenance intervals. Arunraj and Maiti [13] propose the application of AHP (analytic hierarchy process) and goal programming (GP) for maintenance policy selection. Fouladgar et al. [14] use AHP and COPRAS under fuzzy environment to select maintenance strategies.

This paper is structured as follows: An introduction is presented in Section 1. A notation related to multicriteria decision model is presented in Section 2. In Section 3 there is a conceptual background including RCM and MAUT (Multiattribute Utility Theory). The multicriteria model proposed is presented in Section 4. Section 5 presents the application of a multicriteria model based on data from the literature. Some discussions are presented in Section 6. The conclusions of the main results of this paper are presented in Section 7.

\section{Notation}

$A$ : Set of all possible actions under consideration.

$a$ : Constant $(a>0)$ defined by DM for each onedimensional utility function, in order to adjust the information visualization that illustrates his/her attitude with respect to risk.

$a_{i}: i$ th action.

$b$ : Constant $(b>0)$ defined by DM for each onedimensional utility function, in order to adjust the information visualization that illustrates his/her attitude with respect to risk.

$B_{1}$ : Attribute 1 .

$B_{2}$ : Attribute 2.

DRI: Difference ratio index.

(e): Environmental consequence dimension.

$(f)$ : Financial consequence dimension.

(h): Human consequence dimension.

$h_{0}$ : The lowest value of the number of people affected.

$h$ : A value of the number of people affected (between $h_{0}$ and $\left.h_{1}\right)$.

$h_{1}$ : The highest value of the number of people affected.

$k_{1}, k_{2}, k_{3}, k_{4}$, and $k_{5}$ : Scale constants which indicate the value of the trade-off (consequence dimensions).
Lot and Lot': Lotteries.

$\mathrm{MF}_{i}$ : $i$ th failure mode.

$N$ : The adjustment factor of the curve.

$\left(o_{1}\right)$ : Operational 1 consequence dimension.

$\left(o_{2}\right)$ : Operational 2 consequence dimension.

$P\left(q \mid \theta, a_{i}\right)$ : Consequence function.

$q$ : Consequences under study.

$t$ : The downtime.

$U$ : Utility function.

$U(h)$ : The utility function for the human dimension.

$U(e)$ : The utility function for the environmental dimension.

$U(f)$ : The utility function for the financial dimension.

$U\left(o_{1}\right)$ : The utility function for the operational 1 dimension.

$U\left(o_{2}\right)$ : The utility function for the operational 2 dimension.

$U\left(\mathrm{MF}_{i}\right)_{\beta_{i}}$ : Utility of $i$ th failure mode.

$y$ : Represents the values of the consequence considered in a given dimension of consequences.

\section{Greek Symbols}

$\beta_{i}$ : Failure mode ranking position.

$\theta$ : State of nature expressing the uncertainty associated with the problem, representing a failure scenario of a given component.

$\theta^{\prime}$ : No fault occurrence scenario.

$\mu$ : The deviation of $\ln t$.

$\pi(\theta)$ : Probability of each state of nature.

$\sigma:$ The mean of $\ln t$.

\section{Conceptual Background}

The conceptual background consists of two main issues that are integrated into the decision model: a Reliability-Centered Maintenance (RCM) approach and the Multiattribute Utility Theory (MAUT).

3.1. RCM: Reliability-Centered Maintenance. According to Moubray [6], the first industry to systematically confront challenges associated with choices related to maintenance was that of commercial aviation. This resulted in the development of decision-making processes known as MSG3 in aviation and RCM (Reliability-Centered Maintenance) in other sectors.

To Heo et al. [15], RCM is a structured methodology that is used to analyze potential failures of components with a focus on reliability preservation.

For Yssaad et al. [16] and Jiang et al. [17], RCM is a technique applied to develop cost effective maintenance plans, so 
that the capability of the equipment is reached, restored, or maintained. The main RCM goal is to reduce the maintenance cost while focusing on the most relevant functions of the system under study.

Selvik and Aven [8] emphasize that the RCM is a wellestablished method of analysis for planning preventive maintenance, where reliability is the main point of interest. However, failure consequences also should be evaluated. The main RCM goal is to reduce maintenance costs while simultaneously raising reliability and safety. The stages of RCM will be detailed in the next subsection.

3.2. RCM Stages. The divisions of the stages of implementing RCM are considered differently by different authors $[6,18-$ $20]$. Based on these authors, a brief description of the steps of implementing RCM is as follows.

Step 1 (study preparation). Requirements, policies, and acceptance criteria with respect to ensuring safety and protecting the environment should be defined clearly for the RCM analysis.

Step 2 (define system and subsystem boundary). The system is divided into mutually exclusive subsystems which have boundaries that are defined and respected.

Step 3 (identification of functions). At this stage it is necessary to define the functions of each asset within the operational context, together with the desired performance standards.

Step 4 (define functional failures of the assets). At this stage, failures that may occur in the asset should be identified. RCM does this at two levels, namely, by identifying the circumstances that cause a functional failure and secondly through inquiry about what events can cause functional failure of the asset.

Step 5 (define failure modes of each functional failure and the effects). At this stage, events that probably cause each functional failure should be identified. These events are known as failure modes. This step is also important to identify the cause and the effects of every failure.

Step 6 (identify the consequences). At this stage, consequences of failure should be identified.

Step 7 (managing failure). In this step, decisions should be made on what should be done to anticipate, prevent, detect, or correct the failure. This can be verified by applying two techniques for fault management: proactive tasks and default actions.

According to Moubray [6], the process of identifying functions, functional failures, failure modes, and failure effects often leads to opportunities for enhancing performance and security and eliminating waste.

3.3. Multiattribute Utility Theory. Regarding the current world scenario, the decision-making has increasingly become an object of study due to the great importance attached to its consequences.

In this context Vincke [21] observes that Multicriteria Decision Aid (MCDA) is a set of methods and techniques developed to assist organizations and individuals in solving decision problems.

According to Munda [22], as to the operational point of view, the greatest strength of multicriteria decision methods is their ability to look at several points of view, characterized as conflicting issues, which enables an integrated assessment of the problem in question to be made.

For Durbach and Stewart [23], in the decision-making process, the consequences of an action are often unknown due to dependence on future events. Many models are applied under a context of uncertainty, in which some of them are based on MAUT. MAUT provides an evaluation of the probability distribution of each attribute as well as trade-offs involving lotteries.

Keeney and Raiffa [24] state that MAUT is derived from Utility Theory and Decision Theory. According to Alencar and de Almeida [25], the basic idea of Utility Theory is to quantify a decision-maker's (DM's) wishes by relating assets with values that represent a rule of choice for the DM.

Alencar et al. [26] argue that the Utility Theory concepts enable the problem to be converted into an algebraic form that uses the DM's preferences. These preferences are represented by a function named a utility function that associates criteria involved in the problem.

Garcez and de Almeida [27] argue that an important aspect is to identify and appoint the correct DM, given that the decision-making process may involve different types of impacts (operational, human, financial, environmental, etc.). The DM needs to present knowledge and experience about the system taking into consideration aspects such as possible failure, consequences dimensions, and preventive measures.

According to Keeney and Raiffa [24], if an appropriate utility is assigned to each possible consequence and the expected utility of each alternative is calculated, the best course of action is deemed to be the alternative with the highest expected utility.

\section{Multicriteria Decision Model}

The decision model proposed consists of improving the RCM approach by incorporating contributions of a multicriteria decision method. The main objective of the proposed model is to allow a more effective maintenance planning, taking into account the assignment of priorities through the multidimensional analysis of the consequences.

The method chosen is MAUT, which incorporates the necessary probabilistic background for the analysis required and, according to de Almeida et al. [28], it has a well-structured protocol, supported by a very consistent axiomatic framework that could be applied for decisions involving multiple criteria.

The main improvement is given in Step 6 of RCM which is the step that identifies and analyzes the consequences. New steps are introduced, as shown in Figure 1. 


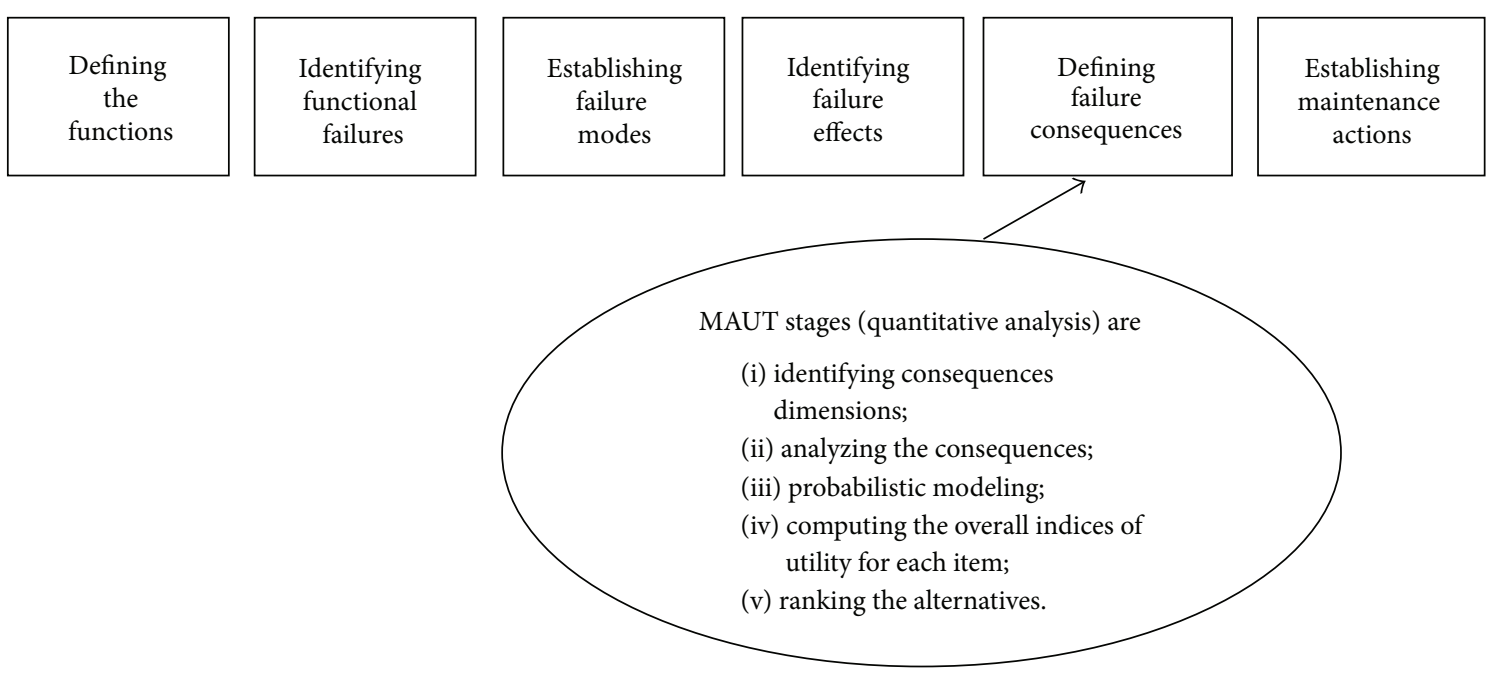

FIgURE 1: Stages of a multicriteria decision model.

4.1. Identifying Consequence Dimensions. According to Carretero et al. [29], since the failures are known, the consequences must be taken into account. These consequences are used as a basis for making strategic decisions related to maintenance. The decision process is used in order to select the activities most suitable for maintaining the system. Hundreds of possible failure modes, of any installation, provoke different effects on safety, the environment, operations, or other costs related to the operations. These consequences are what most influence the extent to which each maintenance action tries to prevent failures. Moubray [6] affirms that a positive point of the RCM is that it recognizes that the consequences of failure are more important than the technical characteristics of the failures themselves.

As noted in $[6,29,30], \mathrm{RCM}$ classifies these consequences into four groups, namely, the consequences of the following:

(i) Hidden failures: they do not have a direct impact but expose the organization to multiple failures with serious, often catastrophic, consequences.

(ii) Safety and environmental failures: a failure has safety consequences such as the fact that people may be killed or injured. The environmental consequences can lead to a violation of some company, regional, national, or even international environmental standard.

(iii) Operational failures: a failure has operational consequences if it affects production.

(iv) Nonoperational failures: evident failures which are in this category did not affect either safety or production but only involve the direct cost of repair.

According to [6], failures affect operations in the following ways: total output, product quality, and customer service are all adversely affected and operating costs plus the direct cost of repair increase.

However, the multicriteria model proposed in this paper assesses the consequences of failures in five dimensions, as shown in Figure 2, where one has the following.
Human Dimension (h). This dimension takes into account deaths, injuries, and other negative outcomes for people affected by the consequences of failure. The estimation is provided based on the number of people affected.

Environmental Dimension $(m)$. This consequence dimension takes into account the area affected as a result of the failure. The measure used is an estimate of the affected area $\left(\mathrm{m}^{2}\right)$ as a consequence of the failure.

Financial Dimension. This takes into account fines paid to customers for delays, dips in performance and in complying with standards, and compensations, besides the direct costs of repair.

Operational 1 Dimension $\left(o_{1}\right)$. This considers the influence of the consequences of the failure on the behavior of the production system. These consequences affect the production process without interrupting the operation. The downtime of the item that presents a failure is used to estimate the consequences.

Operational 2 Dimension $\left(\mathrm{o}_{2}\right)$. This considers the influence of the consequences of the failure on the behavior of the production system. But, in this case, the consequence of failure brings the operation to a halt. The value " 0 " is attributed to the "working" operational status while the value " 1 " is attributed to the "halted" operational status. Downtime is used to estimate the consequences.

4.2. Analysis of the Consequences. To analyze the consequences in this paper, elements of Decision Theory will be used. According to Berger [31], statistical Decision Theory is interested in decision-making in the presence of statistical knowledge and focuses on uncertainties involved in decisionmaking.

Based on Berger [31], a probabilistic approach is used to deal with the uncertainties in $A$ by a probability distribution 


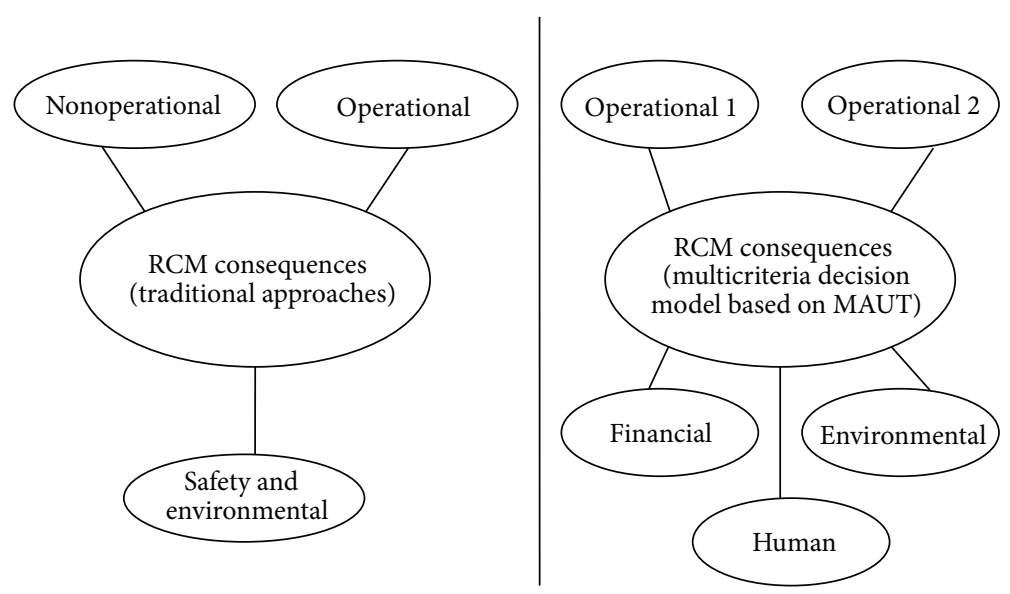

FIgURE 2: Comparison between RCM Consequences (traditional and MCDA approaches).

over deterministic consequences and the elicitation of utility functions for these consequences.

According to Keeney and Raiffa [24], the values of the utilities functions are defined in an interval scale between the extremes $[0,1]$, where " 0 " is associated with the "least preferred" and the extreme " 1 " is associated with the "most preferred."

The utility is calculated by combining the probability of the deterministic consequences " $q$ " in " $A$." Utility is defined according to (1) for discrete cases and by (2) for continuous cases:

$$
\begin{aligned}
& U\left(\theta, a_{i}\right)=\sum_{q} P\left(q \mid \theta, a_{i}\right) U(q), \\
& U\left(\theta, a_{i}\right)=\int_{q} P\left(q \mid \theta, a_{i}\right) U(q) d q .
\end{aligned}
$$

The utility combines the consequence function and utility function on these consequences.

4.3. Probabilistic Modeling. According to Vincke [21] choosing MAUT as a method to be applied is equivalent to choosing a type of compensation among criteria.

The elicitation of utility functions occurs in a closed consequence interval, where a null result (no impact) is associated with maximum utility, while the minimum value of utility is related to the greatest of the consequences estimated.

The objective of this model is to obtain $U$ from an individual or group of individuals whose values are of interest. According to Keeney [32], the approach considered consists of subdividing the assessment of $U$ into parts, working with these parts, and then aggregating them later. This requires the DM's judgment of the final qualitative judgments to be affirmed and quantified.

According to Keeney and Raiffa [24], in intracriteria evaluation, when the evaluation is subdivided into parts of $U$, the curves that depict the DM's preference in relation to the dimensions of impact considered in the model (one-dimensional utility functions) are adjusted. These adjustments take into account the DM's risk behavior. This behavior is incorporated into each $U$ by the nature of the utility function.
Thus, the DM can be prone, averse, or neutral to a given risk situation in each of the dimensions analyzed. These assumptions about the DM's behavior are mutually exclusive and collectively exhaustive when applied to any lottery. Thus, if one wants to compute $U(h)$ for human dimension such that $h_{0} \leq h \leq h_{1}, h$ is a value within this interval. To human dimension, it is assumed that a higher level of people affected is less preferable than a lower level of people affected. To evaluate the DM attitude regarding risk, it is considered a lottery 50-50 (a chance of 50\% to get the consequence $h_{0}$ and a chance of $50 \%$ to get the consequence $h_{1}$ ) where the extremes of $h$ are compared with the expected consequence. Thus, a question is conducted to the DM: Is a 50-50 chance to $h_{0}$ and $h_{1}$ preferable, indifferent, or less preferable than a consequence that will certainly occur $\left(h^{\prime}=\left(h_{0}+h_{1}\right) / 2\right)$ ? A preference for this last consequence, in other words, a preference for the expected value of the lottery, indicates a risk aversion. Thus, the equations used in this model to calculate one-dimensional utility functions take into account the DM's attitudes with respect to risk and are given below:

$$
\begin{aligned}
& U(q)=a-b y, \\
& U(q)=e^{-b y}, \\
& U(q)=1-\left(\frac{y}{\max (y)}\right)^{N} .
\end{aligned}
$$

Equation (3) represents the behavior of the neutrality of decision-making in relation to risk. Equation (4) will be used in situations where the DM is prone to risk. Equation (5) represents the DM's aversion to risk.

Different considerations are established for defining the consequence function in each dimension considered. In the human, environmental, and financial dimensions, consequence functions have been considered to be $P(q \mid \theta, i)=1$ because it is considered that the state of nature is observed and that it is right that these consequences on these dimensions will occur.

For the last two dimensions to be presented $\left(o_{1}\right.$ and $o_{2}$ ), a lognormal probability density function was adopted to 


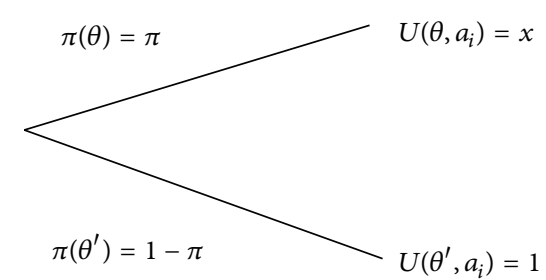

FIGURE 3: How a lottery is applied in order to define utilities in "failure" and "no failure" scenarios.
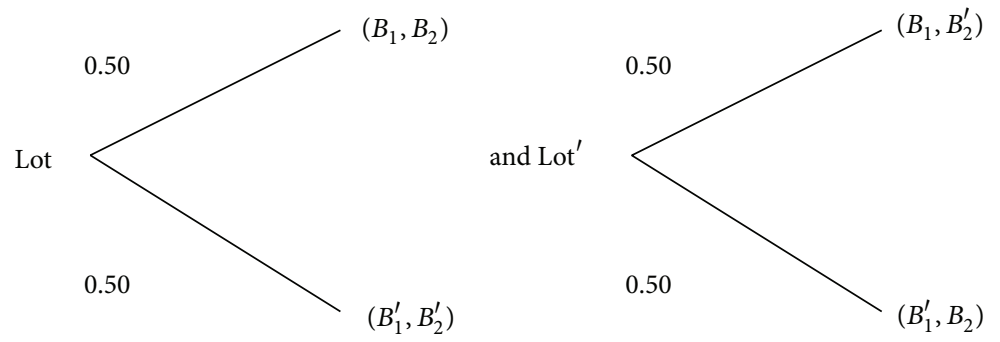

Figure 4: Two lotteries (Lot and Lot') representing the property of additive independence.

calculate the consequence function, where the lognormal is defined by the following equation:

$$
\begin{array}{r}
g(t \mid \mu, \sigma)=\frac{1}{\sigma \cdot t \cdot \sqrt{2 \pi}} \exp \left[-\frac{(\ln t-\mu)^{2}}{2 \sigma^{2}}\right] \\
\text { for }(t \geq 0) .
\end{array}
$$

The choice of this probability distribution is justified by the fact that it represents the downtime adequately. The lognormal distribution describes in a satisfactory way this maintainability situation.

Still regarding intracriteria evaluation, two different situations with respect to failures scenarios that cannot occur simultaneously will be considered. The first possible situation refers to the existence of a failure scenario of a given component, with an occurrence probability $\pi(\theta)=\pi$ and an unidimensional utility $U\left(\theta, a_{i}\right)=x$, with $0 \leq x<1$. The second condition refers to the possible existence of a no fault occurrence scenario with an occurrence probability $\pi\left(\theta^{\prime}\right)=1-\pi$ and a utility $U\left(\theta^{\prime}, a_{i}\right)=1$, in accordance with Figure 3. The sum of these probabilities must be equal to 1 $\left(\pi(\theta)+\pi\left(\theta^{\prime}\right)=1\right)$, where the unidimensional utility $U$ is obtained by

$$
U=U\left(\theta, a_{i}\right) \pi(\theta)+U\left(\theta^{\prime}, a_{i}\right) \pi\left(\theta^{\prime}\right),
$$

where the value of $U(\theta, i)$ set in the interval could be obtained by (1) or (2). The $U\left(\theta^{\prime}, i\right)$ value is always equal to one $U\left(\theta^{\prime}, a_{i}\right)=1$. This is due to the absence of failure always being associated with the "most preferable" situation in the model.

The proposed model seeks obtaining a utility function $U$ of an individual or group of individuals whose values are of interest. The approach taken into account consists of subdividing the $U$ evaluation into parts, working with these parts, and then aggregating them. This requires the DM's judgment of the final qualitative judgments to be affirmed and quantified.

After obtaining both $U$ and $K$ values that are elicited by the elicitation procedure based on the comparison of lotteries [24], the multiattribute utility function is calculated using the equations presented in the following subsection.

4.4. Computing the Overall Indices of Utility. The additive utility function is considered in this model. $B_{1}$ and $B_{2}$ are additively independent if Lot and Lot' ${ }^{\prime}$ are equally preferable for $B_{1}$ and $B_{2}$ and for $B_{1}^{\prime}$ and $B_{2}^{\prime}$ chosen in an arbitrary manner (Figure 4). This means that in each lottery there is a chance of $50 \%$ to obtain $B_{1}$ or $B_{1}^{\prime}$ independently of $B_{2}$ value [24].

This can be represented by the following expression, which gives the independence of the DM's preferences with respect to the five dimensions considered: human $(h)$, environment $(m)$, financial $(f)$, operational $1\left(o_{1}\right)$, and operational $2\left(o_{2}\right)$ :

$$
\begin{aligned}
U\left(h, m, f, o_{1}, o_{2}\right)= & k_{1} U(h)+k_{2} U(m)+k_{3} U(f) \\
& +k_{4} U\left(o_{1}\right)+k_{5} U\left(o_{2}\right) ;
\end{aligned}
$$

$k$ 's are elicited by the procedures of elicitation based on the comparison of lotteries. In this structured process, according to [28] the DM answers some questions, making choices of lotteries considering payoffs between the dimensions considered. The DM should define a probabilistic value $p$ that represents the indifference between the certainty of choice of a consequence (in this case, $p=1$ ) or the choice of a specific lottery. Based on this process, the $p$ value is obtained and consequently $k_{1}=p$. Similar steps are conducted to define $k_{2}, k_{3}, k_{4}$, and $k_{5}$. The sum of $k^{\prime}$ s needs to be equal to " 1 " $\left(k_{1}+k_{2}+k_{3}+k_{4}+k_{5}=1\right)$.

4.5. Ranking the Alternatives. Among different problematics that could be observed, the ranking problematic arises from 
the DM needing to construct a list of the most important alternatives in order of relevance (prioritization), such that the alternatives that are most likely to occur will be primarily treated using all available resources [33]. In the model proposed in this paper the alternatives are represented by failure modes of the system components in analysis. It is only after this initial prioritization that other system failure modes (according to their ranking) are taken into account, since resources are available. MAUT is used to obtain this ranking.

Due to the axiomatic structure of Utility Theory, obtaining another type of information becomes feasible: the ranking of multiattribute utility values. This is obtained from (8).

According to [28], the interval scale of the utility function allows comparison of the incremental value to the failure modes.

Because of this interval scale, we cannot say that the utility associated with a failure mode $\mathrm{MF}_{I}$ is " $Z$ " times greater than the utility associated with a failure mode $\mathrm{MF}_{J}$.

However, it is possible to say that the difference $U\left(\mathrm{MF}_{i}\right)_{\beta_{i}}-U\left(\mathrm{MF}_{\mathrm{j}}\right)_{\beta_{i+1}}$ is " $Z$ " times greater than the difference $U\left(\mathrm{MF}_{j}\right)_{\beta_{i+1}}-U\left(\mathrm{MF}_{k}\right)_{\beta_{i+2}}$. From these explanations the increment ratio of these differences is verified by the DRI:

$$
\mathrm{DRI}=\frac{\left[U\left(\mathrm{MF}_{i}\right)_{\beta_{i}}-U\left(\mathrm{MF}_{j}\right)_{\beta_{i+1}}\right]}{\left[U\left(\mathrm{MF}_{j}\right)_{\beta_{i+1}}-U\left(\mathrm{MF}_{k}\right)_{\beta_{i+2}}\right]}
$$

\section{Numerical Application}

A numerical application is presented in this section in order to illustrate the multicriteria decision model used to evaluate the consequences of failure obtained from the RCM approach. For this application, a realistic production system will be considered in which 18 components considered to be the most relevant will be selected. The existence of only one unique user of this system is also considered.

Five dimensions-human $(h)$, environmental $(e)$, financial $(f)$, operational $1\left(o_{1}\right)$, and operational $2\left(o_{2}\right)$-for estimating the set of consequences were defined. The utility function was considered additive in this application [24], which means that the preference independence of the DMs with respect to these dimensions was verified and taken into account.

For each component $i$ considered in the system, a failure mode $\mathrm{MF}_{i}$ was adopted with its associated occurrence probability $\pi(\theta)_{n}$, as shown in Table 1 .

With the support of a DSS (Decision Support System), consequence probabilities were estimated for the five dimensions of consequences. In the same way, the one-dimensional utility functions are obtained by (3), (4), (5), and (6) taking into account the DM's attitudes with respect to risk. For example, to human dimension, the risk aversion behavior is considered by the DM. On the same way, to financial dimension, the risk prone behavior is defined by the DM. Following an elicitation procedure based on the comparison of lotteries defined in Keeney and Raiffa [24], scale constants are established: $k_{1}=0.19, k_{2}=0.14, k_{3}=0.21, k_{4}=0.18$, and $k_{5}=0.28$.
TABLE 1: Probabilities of failure modes for each component.

\begin{tabular}{lcc}
\hline Component “ $i$ " & Failure $\mathrm{mode} \mathrm{MF}_{i}$ " & “ $\pi(\theta)_{i}$ ” \\
\hline 1 & $\mathrm{MF}_{1}$ & 0.0356 \\
2 & $\mathrm{MF}_{2}$ & 0.0658 \\
3 & $\mathrm{MF}_{3}$ & 0.0752 \\
4 & $\mathrm{MF}_{4}$ & 0.0211 \\
5 & $\mathrm{MF}_{5}$ & 0.0653 \\
6 & $\mathrm{MF}_{6}$ & 0.0200 \\
7 & $\mathrm{MF}_{7}$ & 0.0550 \\
8 & $\mathrm{MF}_{8}$ & 0.0188 \\
9 & $\mathrm{MF}_{9}$ & 0.0854 \\
10 & $\mathrm{MF}_{10}$ & 0.0365 \\
11 & $\mathrm{MF}_{11}$ & 0.0698 \\
12 & $\mathrm{MF}_{12}$ & 0.0150 \\
13 & $\mathrm{MF}_{13}$ & 0.0442 \\
14 & $\mathrm{MF}_{14}$ & 0.0952 \\
15 & $\mathrm{MF}_{15}$ & 0.0325 \\
16 & $\mathrm{MF}_{16}$ & 0.0666 \\
17 & $\mathrm{MF}_{17}$ & 0.0482 \\
18 & $\mathrm{MF}_{18}$ & 0.0140 \\
\hline
\end{tabular}

Using these values, the multiattribute utility function $U\left(h, e, f, o_{1}, o_{2}\right)$ must be elicited from the DM, following the established procedures defined in Keeney and Raiffa [24]. These procedures are structured protocols that take into account choices between consequences of functional failures and lotteries with specific probabilities between the best and worst case.

A conversion scale is introduced into the values of the multiattribute utility function to facilitate the interpretation of these values. The axiomatic structure of Utility Theory allows another type of feasible information: the ranking of multiattribute utility values for each failure mode as considered in Table 2.

The interval scale [28] of the utility functions permits comparisons of the increments of utility in relation to failure modes. These increments are shown in Table 3.

From this data analysis, failure mode $\mathrm{MF}_{18}$ should be the first to receive an allocation of the resources available in the company.

On analyzing the values in Table 3 , it can be verified that the difference between the values of the utilities associated with failure modes $\mathrm{MF}_{03}$ and $\mathrm{MF}_{11}$ is 0.129727 and that the difference between the values of the utilities associated with failure modes $\mathrm{MF}_{11}$ and $\mathrm{MF}_{17}$ is 0.006064 . This means that an increment in the utility values from $M_{03}$ to $M F_{11}$ is approximately 21.4 times greater than the increment in the utility values from $\mathrm{MF}_{11}$ to $\mathrm{MF}_{17}$. This analysis is based on the interval scale provided by the utility function. In the same way, it can be verified that the difference between the values of the utilities associated with the failure modes $\mathrm{MF}_{17}$ and $\mathrm{MF}_{02}$ is 0.0122 and that the difference between the values of the utilities associated with the failure modes $\mathrm{MF}_{02}$ and $\mathrm{MF}_{05}$ is 0.000043 . This means that an increment in the utility values from $\mathrm{MF}_{17}$ to $\mathrm{MF}_{02}$ is approximately 285 times greater than 
TABLE 2: Utility ranking of the failure modes resulting from Table 1.

\begin{tabular}{lcc}
\hline Ranking position $\left(\beta_{i}\right)$ & Failure $\operatorname{mode} \mathrm{MF}_{i} "$ & $U\left(\mathrm{MF}_{i}\right)_{\beta_{i}}$ \\
\hline$\beta_{1}$ & $\mathrm{MF}_{18}$ & 0 \\
$\beta_{2}$ & $\mathrm{MF}_{12}$ & 0.19256 \\
$\beta_{3}$ & $\mathrm{MF}_{09}$ & 0.43039 \\
$\beta_{4}$ & $\mathrm{MF}_{16}$ & 0.48879 \\
$\beta_{5}$ & $\mathrm{MF}_{03}$ & 0.58785 \\
$\beta_{6}$ & $\mathrm{MF}_{11}$ & 0.71757 \\
$\beta_{7}$ & $\mathrm{MF}_{17}$ & 0.72364 \\
$\beta_{8}$ & $\mathrm{MF}_{02}$ & 0.73584 \\
$\beta_{9}$ & $\mathrm{MF}_{05}$ & 0.73588 \\
$\beta_{10}$ & $\mathrm{MF}_{07}$ & 0.74578 \\
$\beta_{11}$ & $\mathrm{MF}_{14}$ & 0.75274 \\
$\beta_{12}$ & $\mathrm{MF}_{13}$ & 0.82960 \\
$\beta_{13}$ & $\mathrm{MF}_{01}$ & 0.88966 \\
$\beta_{14}$ & $\mathrm{MF}_{10}$ & 0.91708 \\
$\beta_{15}$ & $\mathrm{MF}_{15}$ & 0.92116 \\
$\beta_{16}$ & $\mathrm{MF}_{04}$ & 0.96991 \\
$\beta_{17}$ & $\mathrm{MF}_{06}$ & 0.98484 \\
$\beta_{18}$ & $\mathrm{MF}_{08}$ & 1 \\
\hline
\end{tabular}

the increment in the utility values from $\mathrm{MF}_{02}$ to $\mathrm{MF}_{05}$. These increments of utility in relation to failure modes provide to decision-maker a differentiated interpretation of results, providing an information visualization that allows a better resources allocation to prevent and mitigate failure modes.

\section{Discussion}

Many of the existing traditional approaches applied to quantitative consequences of failures analysis in the industrial environment do not incorporate a multidimensional assessment of the problem. Thus, this type of evaluation is performed without considering the real impact that consequences may entail in multiple dimensions that should be assessed in the context under study. This issue worsens as the complexity of the context increases, where relevant aspects are not considered since the analysis includes only the study of a single dimension. However, it is important to emphasize that it is ever more important for an organization to assess possible issues associated with the occurrence of failures such as injuries to people or the number of fatalities, financial losses, environmental impact, damage to properties, and solving problems with different factors [34]. This occurs due to the fact that in the real world the management of consequences needs to satisfy a number of expectations of the various stakeholders.

Therefore, the issues discussed in this section justify the use of multicriteria approaches to manage the consequences of failures in industrial environments, such that the decision process is developed in a coherent way, considering the consequence dimensions judged as being the most relevant to the context under study.
Among the set of techniques and methods available for multicriteria evaluation, MAUT was chosen for the development of this model, since it presents some important specific features that enable the uncertainty in the environment to be dealt with in a more appropriate way, based on MAUT's axiomatic structure, taking into account probabilistic aspects and considering the DM's value judgments, based on his/her preferences.

Another very important aspect of MAUT can be seen in the step for ranking the alternatives (failure modes). The calculation of the difference ratio index (DRI) enables a differentiated management analysis by the DM, thereby adding important aspects of the decision process and allowing some new information to be perceived. The calculation is defined as the ratio of absolute differences between pairs of failure modes arranged in order of ranking, taking into account values established in the calculation of multiattribute utility functions for each failure mode considered. More specifically, the absolute difference that comprises the DRI is determined by a subtraction of multiattribute utilities values linked with two subsequent failure modes in the final ranking obtained. The DRI is calculated for all failure modes (pairwise comparison) that comprise the ranking. This type of analysis is only possible due to a specific feature of the utility function, namely, the interval scale. This type of analysis supports the understanding of different levels of failure modes and their criticality being evaluated, thereby making it clear that there is a real need for resources, which are often scarce, to be allocated. This occurs through larger or smaller volumes of investment, depending on the gravity of the scenario observed. Based on the ranking obtained, the manager can define how much and where to invest in order to minimize or even eliminate the impacts of the consequences. This investment takes place through targeted management actions for the maintenance, operation, monitoring, and control of planned actions. Thus, what is sought as a final process result is to use the information obtained from a ranking as input so that managerial decisions are made in the best possible way, taking into account the analysis of all the dimensions considered in the model.

Finally, it is important to emphasize that a sensitivity analysis was conducted based on the data and parameters analyzed to verify the robustness of the model. Results show that the procedure was robust.

\section{Conclusions}

The appropriate allocation of investments given the reality of resources being scarce requires maintenance managers to have pertinent knowledge and to use adequate tools to allocate resources in maintenance actions in a better way. The RCM approach is widely used in this sense, thereby ensuring that assets continue to perform their functions within the operating environment. The use of a multicriteria decision model to evaluate quantitatively the consequences obtained from an RCM approach is proposed in this paper in order to ensure an improvement in this process. The model is applied in this context with the purpose of supporting management decisions in an environment of uncertainty on which 
TABLE 3: Comparisons of utility values and ratios of the increments of utility between ranked failure modes.

\begin{tabular}{lccc}
\hline Ranking position $\left(\beta_{i}\right)$ & Failure mode $\mathrm{MF}_{i}$ ” & $U\left(\mathrm{MF}_{i}\right)_{\beta_{i}}-U\left(\mathrm{MF}_{j}\right)_{\beta_{i+1}}$ & DRI \\
\hline$\beta_{1}$ & $\mathrm{MF}_{18}$ & 0.192562 & 0.80968 \\
$\beta_{2}$ & $\mathrm{MF}_{12}$ & 0.237825 & 4.072049 \\
$\beta_{3}$ & $\mathrm{MF}_{09}$ & 0.058404 & 0.589616 \\
$\beta_{4}$ & $\mathrm{MF}_{16}$ & 0.099055 & 0.763565 \\
$\beta_{5}$ & $\mathrm{MF}_{03}$ & 0.129727 & 21.394127 \\
$\beta_{6}$ & $\mathrm{MF}_{11}$ & 0.006064 & 0.497029 \\
$\beta_{7}$ & $\mathrm{MF}_{17}$ & 0.0122 & 284.84424 \\
$\beta_{8}$ & $\mathrm{MF}_{02}$ & 0.000043 & 0.004327 \\
$\beta_{9}$ & $\mathrm{MF}_{05}$ & 0.009899 & 1.421064 \\
$\beta_{10}$ & $\mathrm{MF}_{07}$ & 0.006966 & 0.090634 \\
$\beta_{11}$ & $\mathrm{MF}_{14}$ & 0.076856 & 1.279671 \\
$\beta_{12}$ & $\mathrm{MF}_{13}$ & 0.060059 & 2.190339 \\
$\beta_{13}$ & $\mathrm{MF}_{01}$ & 0.02742 & 6.712401 \\
$\beta_{14}$ & $\mathrm{MF}_{10}$ & 0.004085 & 0.083794 \\
$\beta_{15}$ & $\mathrm{MF}_{15}$ & 0.04875 & 3.266711 \\
$\beta_{16}$ & $\mathrm{MF}_{04}$ & 0.014923 & 0.984148 \\
$\beta_{17}$ & $\mathrm{MF}_{06}$ & 0.015164 & - \\
$\beta_{18}$ & $\mathrm{MF}_{08}$ & - & - \\
\hline
\end{tabular}

the maintenance sector is inserted. The utility function is obtained for each of the failure modes considered. The DM's preferences are taken into account so that the final result for each failure mode incorporates subjective aspects based on the DM's perceptions and behavior. The model provides a great contribution to the phase of consequence analysis. Consequence groups established in the RCM approach are restructured into new five dimensions. The consequences that affect safety and the environment were separated into two new dimensions in order to generate more accurate assessments, given that environmental questions and lives have great relevance to society today.

The implementation of these improvements by using a multicriteria decision model seeks to generate consistent results that can assist managers in their planning of maintenance.

\section{Conflict of Interests}

The authors declare that there is no conflict of interests regarding the publication of this paper.

\section{Acknowledgment}

This paper is part of a research study funded by the Brazilian National Research Council (CNPq).

\section{References}

[1] H. Chouikhi, A. Khatab, and N. Rezg, "A condition-based maintenance policy for a production system under excessive environmental degradation," Journal of Intelligent Manufacturing, vol. 25, no. 4, pp. 727-737, 2014.
[2] J. E. Ruiz-Castro, "Preventive maintenance of a multi-state device subject to internal failure and damage due to external shocks," IEEE Transactions on Reliability, vol. 63, no. 2, pp. 646660, 2014.

[3] C. A. V. Cavalcante and A. T. de Almeida, "A multi-criteria decision-aiding model using PROMETHEE III for preventive maintenance planning under uncertain conditions," Journal of Quality in Maintenance Engineering, vol. 13, no. 4, pp. 385-397, 2007.

[4] A. Ishizaka and P. Nemery, "Assigning machines to incomparable maintenance strategies with ELECTRE-SORT," Omega, vol. 47, pp. 45-59, 2014.

[5] B. Liu, Z. Xu, M. Xie, and W. Kuo, "A value-based preventive maintenance policy for multi-component system with continuously degrading components," Reliability Engineering \& System Safety, vol. 132, pp. 83-89, 2014.

[6] J. Moubray, Reliability-Centered Maintenance, Industrial Press, South Norwalk, NC, USA, 2nd edition, 1997.

[7] N. Z. Kontrec, G. V. Milovanović, S. R. Panić, and H. Milošević, "A reliability-based approach to nonrepairable spare part forecasting in aircraft maintenance system," Mathematical Problems in Engineering, vol. 2015, Article ID 731437, 7 pages, 2015.

[8] J. T. Selvik and T. Aven, "A framework for reliability and risk centered maintenance," Reliability Engineering and System Safety, vol. 96, no. 2, pp. 324-331, 2011.

[9] Y. Wang, C. Deng, J. Wu, Y. Wang, and Y. Xiong, "A corrective maintenance scheme for engineering equipment," Engineering Failure Analysis, vol. 36, pp. 269-283, 2014.

[10] F. T. S. Chan and A. Prakash, "Maintenance policy selection in manufacturing firms using the fuzzy MCDM approach," International Journal of Production Research, vol. 50, no. 23, pp. 7044-7056, 2012.

[11] G. Kumar and J. Maiti, "Modeling risk based maintenance using fuzzy analytic network process," Expert Systems with Applications, vol. 39, no. 11, pp. 9946-9954, 2012. 
[12] A. T. de Almeida, "Multicriteria model for selection of preventive maintenance intervals," Quality and Reliability Engineering International, vol. 28, no. 6, pp. 585-593, 2012.

[13] N. S. Arunraj and J. Maiti, "Risk-based maintenance policy selection using AHP and goal programming," Safety Science, vol. 48, no. 2, pp. 238-247, 2010.

[14] M. M. Fouladgar, A. Lashgari, Z. Turskis, A. Yazdani-Chamzini, and E. K. Zavadskas, "Maintenance strategy selection using AHP and COPRAS under fuzzy environment," International Journal of Strategic Property Management, vol. 16, no. 1, pp. 85104, 2012.

[15] J. H. Heo, M. K. Kim, and J. K. Lyu, "Implementation of Reliability-Centered Maintenance for transmission components using Particle Swarm Optimization," International Journal of Electrical Power and Energy Systems, vol. 55, pp. 238-245, 2014.

[16] B. Yssaad, M. Khiat, and A. Chaker, "Reliability centered maintenance optimization for power distribution systems," International Journal of Electrical Power and Energy Systems, vol. 55, pp. 108-115, 2014.

[17] X. Jiang, F. Duan, H. Tian, and X. Wei, "Optimization of reliability centered predictive maintenance scheme for inertial navigation system," Reliability Engineering \& System Safety, vol. 140, pp. 208-217, 2015.

[18] G. A. de A. Alves, C. A. V. Cavalcante, and R. S. Lopes, "Maintenance management: a study of reliability-centered maintenance for irrigation system," Applied Engineering in Agriculture, vol. 31, no. 2, pp. 227-235, 2015.

[19] P. Dehghanian, M. Fotuhi-Firuzabad, F. Aminifar, and R. Billinton, "A comprehensive scheme for reliability centered maintenance in power distribution systems-part I: methodology," IEEE Transactions on Power Delivery, vol. 28, no. 2, pp. 761-770, 2013.

[20] C. S. Carlson, Effective FMEAs. Achieving Safe, Reliable, and Economical Products and Processes using Failure Mode and Effects Analysis, Wiley Series in Quality \& Reliability Engineering, John Wiley \& Sons, Hoboken, NJ, USA, 1st edition, 2012.

[21] P. Vincke, Multicriteria Decision-Aid, John Wiley \& Sons, Brussels, Belgium, 1992.

[22] G. Munda, Social Multi-Criteria Evaluation for a Sustainable Economy, Springer, Berlin, Germany, 2008.

[23] I. N. Durbach and T. J. Stewart, "A comparison of simplified value function approaches for treating uncertainty in multicriteria decision analysis," Omega, vol. 40, no. 4, pp. 456-464, 2012.

[24] R. L. Keeney and H. Raiffa, Decisions With Multiple Objectives: Preferences and Value Trade-Offs, John Wiley \& Sons, New York, NY, USA, 1976.

[25] M. H. Alencar and A. T. de Almeida, "Assigning priorities to actions in a pipeline transporting hydrogen based on a multicriteria decision model," International Journal of Hydrogen Energy, vol. 35, no. 8, pp. 3610-3619, 2010.

[26] L. H. Alencar, C. M. D. M. Mota, and M. H. Alencar, "The problem of disposing of plaster waste from building sites: problem structuring based on value focus thinking methodology," Waste Management, vol. 31, no. 12, pp. 2512-2521, 2011.

[27] T. V. Garcez and A. T. de Almeida, "A risk measurement tool for an underground electricity distribution system considering the consequences and uncertainties of manhole events," Reliability Engineering and System Safety, vol. 124, pp. 68-80, 2014.

[28] A. T. de Almeida, C. A. V. Cavalcante, M. H. Alencar, R. J. P. Ferreira, A. T. de Almeida-Filho, and T. V. Garcez, Multicriteria and Multiobjective Models for Risk, Reliability and Maintenance Decision Analysis, vol. 231 of International Series in Operations Research \& Management Science, Springer, New York, NY, USA, 2015.

[29] J. Carretero, J. M. Pérez, F. García-Carballeira et al., "Applying RCM in large scale systems: a case study with railway networks," Reliability Engineering and System Safety, vol. 82, no. 3, pp. 257273, 2003.

[30] I. B. Hipkin and A. G. Lockett, "A study of maintenance technology implementation,” Omega, vol. 23, no. 1, pp. 79-88, 1995.

[31] J. O. Berger, Statistical decision theory and Bayesian analysis, Springer Series in Statistics, Springer, New York, NY, USA, 2nd edition, 1985.

[32] R. Keeney, Value-Focused Thinking: A Path to Creative DecisionMaking, Harvard University Press, London, UK, 1992.

[33] T. V. Garcez and A. T. De Almeida, "Multidimensional risk assessment of manhole events as a decision tool for ranking the vaults of an underground electricity distribution system," IEEE Transactions on Power Delivery, vol. 29, no. 2, pp. 624-632, 2014.

[34] W.-C. Chen, L.-Y. Wang, and M.-C. Lin, "A hybrid MCDM model for new product development: applied on the Taiwanese $\mathrm{LifePO}_{4}$ Industry," Mathematical Problems in Engineering, vol. 2015, Article ID 462929, 15 pages, 2015. 


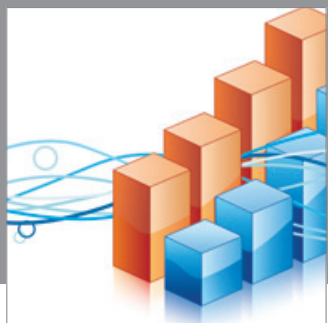

Advances in

Operations Research

mansans

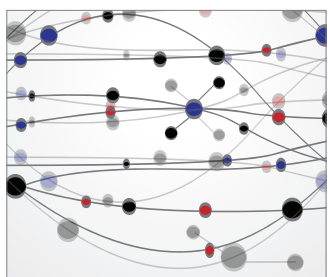

The Scientific World Journal
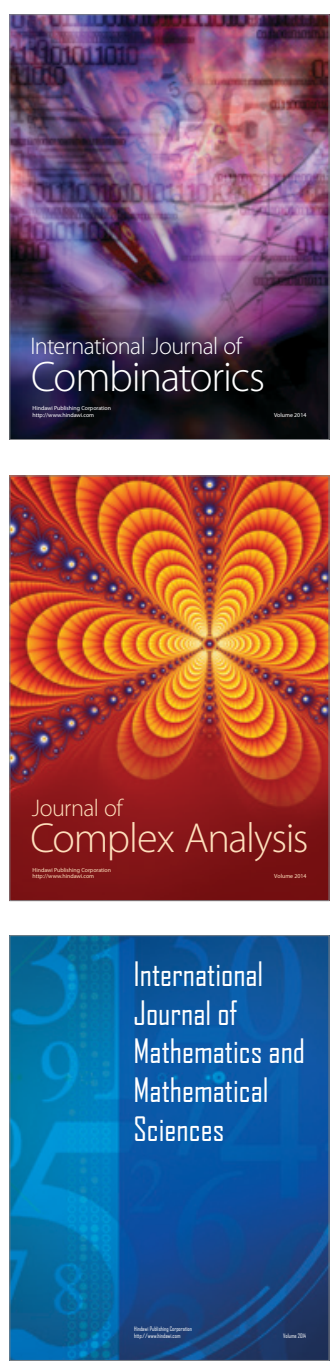
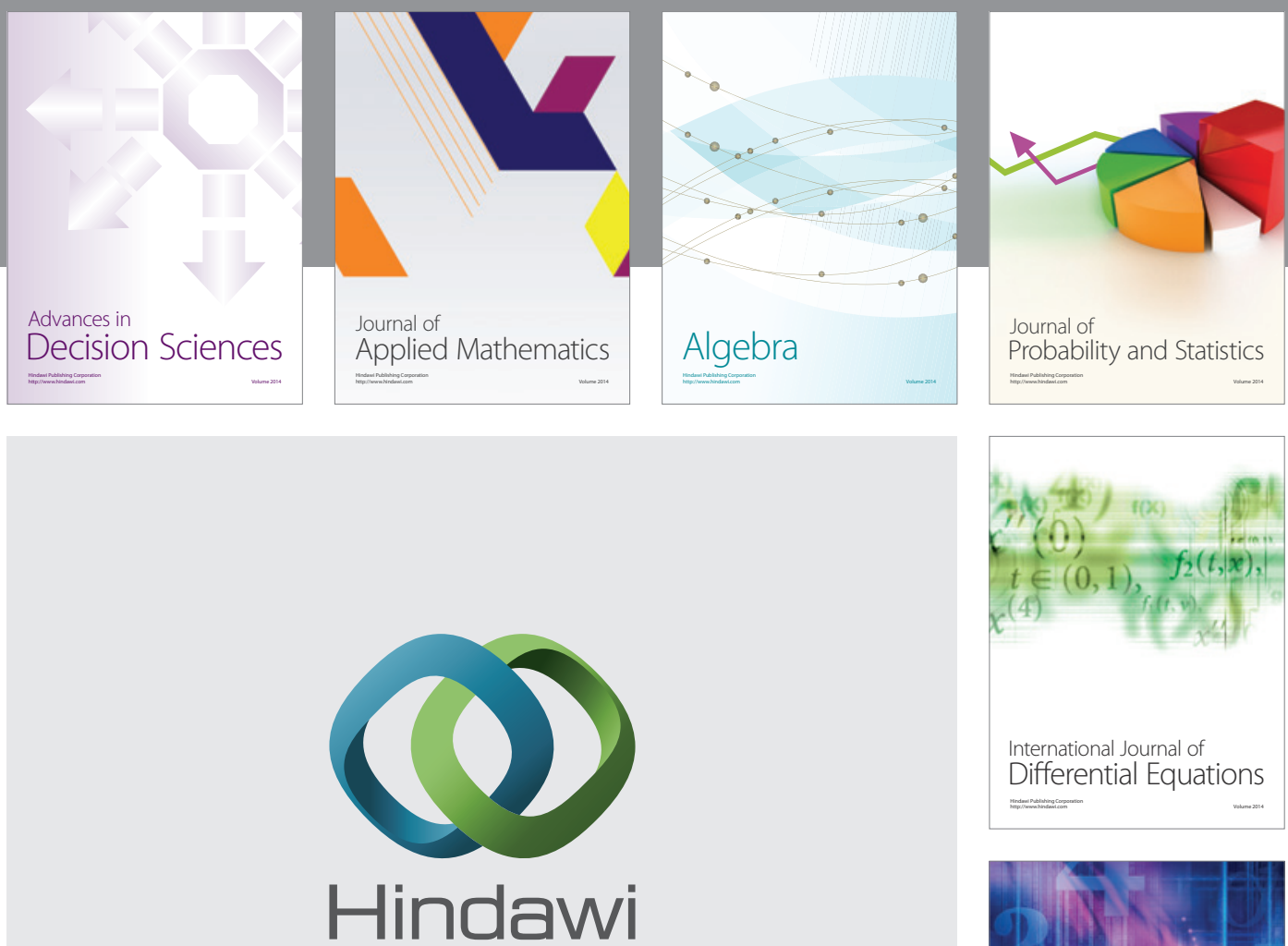

Submit your manuscripts at http://www.hindawi.com
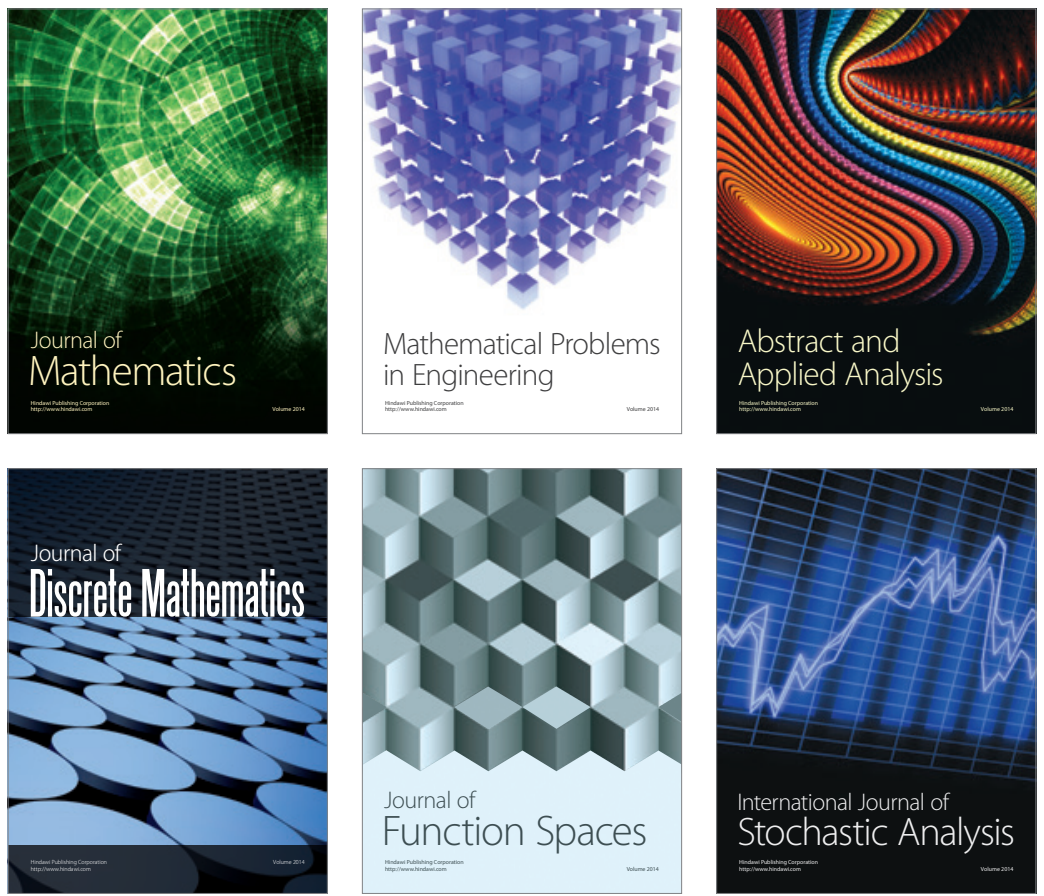

Journal of

Function Spaces

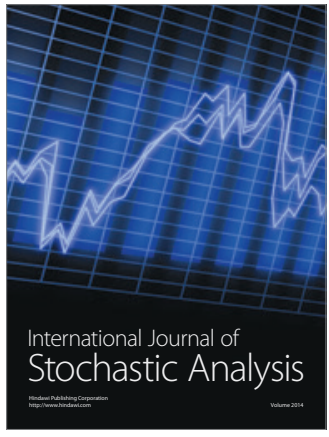

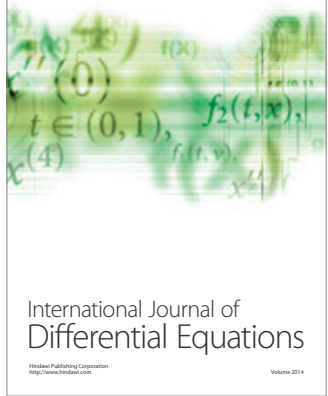
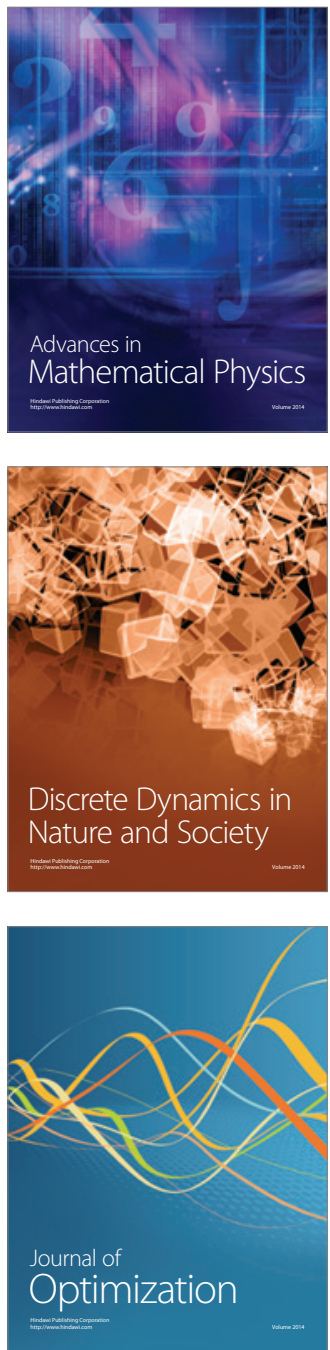